\section{Estudio bibliométrico de la producción científica sobre las TIC en la educación en tiempos de pandemia}

Bibliometric study of scientific production on ICT in education in times of pandemic

\author{
Mamani Ticona, Marìa Isabel* \\ Padilla Caballero, Jesús Emilio Agustín*
}

\section{RESUMEN}

Las TIC en el contexto educativo en tiempos de la pandemia es un tema de gran actualidad en nuestro país, asunto temático que se halla en pleno desarrollo, y por ende, se halla poco estudiado. Descripción comparativa de la producción científica de habla español sobre las TICS entorno a la educación en las bases de datos Scielo, Dialnet y Redalyc durante el 2019 y 2020. Es estudio bibliométrico, descriptivo comparativo y retrospectivo, con una muestra final de 50 artículos de investigación, distribuidos en 25 artículos de trabajos publicados en el 2019 y 25 artículos en el 2020, en revistas indexadas de habla en español en las bases de datos señalados previamente. Se identificó que el país de mayor producción de artículos sobre las TIC con enfoques educativos en habla español, ha sido Ecuador, país que produjo en el 2020 (10\%) en comparación al 2019 (5\%). A La comparación de la producción de artículos de investigación en el 2020, resulta aún incipiente ello, para tener un sustento teórico más detallado y sólido en la idea de aportar estrategias y acciones en la mejora del desarrollo de las TIC con enfoques educativos.

\footnotetext{
* Docente: Bachiller en Ciencias de la Educación Primaria. Segunda especialidad en Educación inicial. Magister en Administración de la Educación, Docente de Educación Superior. Instituto Superior Pedagógico Privado Nueva Esperanza. Correo electrónico institucional mmamanit@nuevaesperanza.edu.pe. Docente de Educación Primaria. Institución Educativa 7079 Ramiro Priale Priale. ORCID: 0000-0002-8020-II9X.

* Docente, Maestro en docencia y gestión educativa, Segunda especialidad en Evaluación de la calidad de los aprendizajes, Doctor en administración de la educación, PhD. en Ética y Responsabilidad Social y derecho Humanos, Docente Investigador y Temático, Universidad César Vallejo, Correo electrónico institucional: jpadillac@ucv.edu.pe, Correo electrónico personal: claimvenst2@yahoo.com, ORCID ID: 0000-0002-97568772
}

\section{REVISTATECNOLÓGICA ciencia y educación Edwards Deming}

ISSN: 2600-5867

\section{Atribución/Reconocimiento-NoCo} mercial- Compartirlgual 4.0 Licencia Pública Internacional — CC

\section{BY-NC-SA 4.0}

https://creativecommons.org/licenses /by-nc- sa/4.0/legalcode.es

Editado por: Tecnológico Superior Corporativo Edwards Deming

Enero - Julio Vol. 5 - I - 202 I

https://revista-edwardsdeming.com/index.php/es e-ISSN: 2576-097I

Recibido: 04 Marzo 2020

Aprobado: II Diciembre, 2020

Pag 57-64 
Palabras clave: Software didáctico, Las TIC y la educación

\section{ABSTRACT}

ICT in the educational context in times of the pandemic, is a very topical issue in our country, a thematic issue that is in full development, and therefore, little studied. Comparative description of the Spanish-speaking scientific production around ICTs around education in the Scielo, Dialnet and Redalyc databases during 2019 and 2020. It is a bibliometric, descriptive, comparative and retrospective study, with a final sample of 50 research articles, distributed in 25 articles of works published in 2019 and 25 articles in 2020, in indexed journals speaking in Spanish in the previously mentioned databases. It was identified that the country with the highest production of articles on ICT with educational approaches in Spanish speaking has been Ecuador, a country that produced in 2020 (10\%) compared to 2019 (5\%). A The comparison of the production of research articles in 2020, it is still incipient, to have a more detailed and solid theoretical support in the idea of providing strategies and actions in improving the development of ICT with educational approaches.

Keywords: Didactic software, ICT and education

\section{INTRODUCCIÓN}

La pandemia del Covid 19, ha creado un nuevo contexto histórico, "un antes y un después" en todos los ámbitos de desarrollo del ser humano, nuevo contexto que ha abierto una oportunidad de desarrollo para las tecnologías de la información y comunicación (TIC), específicamente, en el ámbito de la educación.

Según la UNESCO, I I 3 países han cerrado sus centros educativos para proteger la salud de las y los estudiantes a raíz de la pandemia global. Ante este panorama, el desafío al que se enfrentan los sistemas educativos es el de adaptarse rápidamente al mundo digital (Fundación Wiesse, 2020).

Banco Mundial (2016), señaló que "existe un buen porcentaje de estudiantes que no cuentan con dispositivos electrónicos que les permita ser beneficiados en el uso de las TIC, es decir que unos 4000 millones de estudiantes no cuentan con internet y nuevas tecnologías para adquirir conocimientos, representado el $60 \%$ de la población mundial" (p.66). Los teléfonos móviles y otras tecnologías digitales, se encuentran en auge de alguna manera desarrollando el mundo, podemos encontrar hogares que no cuentan con agua y servicios higiénicos, pero cuentan con un teléfono móvil con internet, esta es la oportunidad de lograr enseñanza aprendizaje. Del mismo modo, el Banco Mundial. (20I4), añadió que "ellos fomentan el desarrollo en muchas regiones del mundo, para mejorar la competitividad educativa de los sectores pobres haciendo que ellos tengan mayor productividad académica" (p.86).

En América Latina, investigadores, docentes, alumnos y el equipo administrativo que da soporte a sus actividades han dicho al mundo que la formación no se detiene, que el aprendizaje no se posterga; por ello, en un esfuerzo titánico han implementado estrategias de educación virtual en tan solo unas semanas. "Es cierto que existen 
aspectos de la formación personal que será muy difícil trasladar a la modalidad en línea; sin embargo, es imperativo en este contexto migrar a una educación no presencial" (UDEP, 2020).

Gracias a las TIC los docentes pueden cumplir con el teletrabajo que les ha sido asignado debido a la emergencia sanitaria y se han visto forzados a dejar los tradicionalismos y poner en práctica nuevas formas de aprendizaje, que como indica García (2020): es mediante la educación a distancia conectados en línea, lo que ha cambiado la forma de educar; esta educación a distancia de probada calidad y eficacia, ha creado una multitud de modelos organizativos, tecnológicos y pedagógicos para una enseñanza y aprendizaje en base a una comunicación y colaboración ubicuas, instantáneas, y sostenidas en tiempo real, lo que en tiempos antiguos era impensable ya que solo era válida la educación presencial donde se utilizaban textos físicos memorísticos.

En lo que concierne a Perú, el gobierno ha emprendido acciones inmediatas con el lanzamiento de la estrategia Aprendo en Casa, la cual incluye una plataforma virtual integrada, así como programación diaria televisiva en señal abierta, y contenido en más de 50 canales de radio que transmiten en más de 10 lenguas originarias. Perú tiene alrededor de 6 millones de estudiantes y el mayor reto que se presenta, desafío común para todos los países, es el acceso para los alumnos en zonas más rurales. Un análisis realizado en el país demuestra que también existe una brecha significativa de conectividad.

Según Castells (2000), las TIC son "un conjunto convergente de tecnologías de la microelectrónica, la informática (máquinas y software), las telecomunicaciones y la optoelectrónica" (P. 66), de otra parte Cabero (1998) plantea :

En líneas generales podríamos decir que las nuevas tecnologías de la información y comunicación son las que giran en torno a tres medios básicos: la informática, la microelectrónica y las telecomunicaciones; pero giran, no sólo de forma aislada, sino lo que es más significativo de manera interactiva e interconexionadas, lo que permite conseguir nuevas realidades comunicativas. (P.67)

Las formas de enseñar han evolucionado, gracias a las TIC y los paradigmas han ido adoptando nuevas estrategias, como la innovación en la pedagogía, por medio de las (TIC) y las Tecnologías para el Aprendizaje y Conocimiento (TAC) ya que los estudiantes viven en la sociedad red, donde ocurren gran parte de los aprendizajes, esto durante la pandemia a resultado un oasis para estudiantes $y$ docentes que desean seguir aprendiendo, donde se aprovecha las múltiples ventajas de la tecnología para formar a los cyber-estudiantes, donde el aula (espacio físico) es un mito, ya que la educación virtualizada es el auge y éxito de los centros educativos, basados en experimentos y simulaciones, gracias a las herramientas tecnológicas desarrolladas, para organizar contenidos, evaluaciones y realizar control de tareas.

Es necesario estar preparados para enfrentar los cambios que exigen las nuevas modalidades de aprendizaje, así como el poner en práctica los conocimientos sobre las nuevas tecnologías (TIC), para Pinedo (20I7): “el acceso a una educación de calidad, es dotar al docente de herramientas que permitan complementar al contenido curricular 
con las tecnologías y herramientas disponibles" (p.78), las cuales son un apoyo para poder presentar una clase diferente, creativa, dinámica, participativa, constructivista, e inclusivo para las estudiantes con discapacidad, de esta forma se despierta el interés de los participantes por aprender; así pues, es importante capacitar a los docentes en (TAC) para que cada educador pueda utilizar diferentes programas en sus clases, para hacerlo de forma interactiva; $y$ podrá configurar el aula virtual de la especialidad que imparte con el objetivo de estar acorde con la tecnología.

Como dicen Cueva, García y Martínez (2019):

La formación educativa de los estudiantes permite el uso adecuado de las TIC y mejora los resultados educativos, por lo que es fundamental la capacitación docente, en donde algunos están conscientes de que no están preparados para vivir en el mundo de la web 2.0, ya que no recibieron los conocimientos necesarios en su formación docente, pero buscan capacitarse para poder educar a los nativos tecnológicos y no quedarse atrás de ellos. (p.55)

Es necesario diferenciar los grupos de personas que utilizan tecnologías, los nativos e inmigrantes digitales, acorde con Prensky (2010):

Los primeros son los que nacieron en un mundo tecnológico utilizando la "lengua digital", mientras en el segundo caso no, pero tratan de adaptarse al entorno y al ambiente, aunque conservando la conexión con el pasado Los llamados nativos digitales no le temen a la tecnología, les gusta y cada día siguen aprendiendo por intuición mediante el ensayo error, dejando a sus docentes en desventaja generando sentimientos de incapacidad. (p.66)

De acuerdo con, Alva (20I5a):

Uno de los retos a superar es las barreras de uso, que es la forma en la que las personas aprovechan al máximo las TIC y las ponen en práctica según las necesidades; hay cantidad de personas sobre todo los inmigrantes digitales que tienen dificultades para acceder a estas nuevas tecnologías trayendo por consiguiente la pobreza digital que se conceptualiza como la carencia en el uso y el acceso de las (TIC). (p.99)

Teniendo en cuenta la escasa producción científica en torno a las TIC enfocados a la educación en tiempos de pandemia, el objetivo de la presente investigación es realizar una análisis descriptivo comparativo de los años 2019 y 2020 de la producción científica de las TIC en torno a la educación, por medio de un estudio bibliométrico, para poder establecer nuevas líneas de investigación en la búsqueda de la calidad de la educación con ayuda de la tecnología.

\section{MATERIALES Y MÉTODOS}

El estudio de investigación se desarrolló por medio de un estudio bibliométrico, de enfoque cuantitativo, nivel descriptivo-comparativo e retrospectivo, teniendo como base teórica las revistas publicadas sobre las TIC en el contexto educativo, con el objetivo de analizar, describir y comparar la producción de revistas de investigación 
durante el 2019 y el 2020, en torno a las publicaciones sobre las TIC en torno a la educación.

La muestra final estuvo constituida por 50 artículos investigación; distribuidos de la siguiente manera: 25 artículos publicados en el año 2020 y, 25 artículos publicados en el año 2019; en revistas indexadas de las bases de datos de Redalyc, Scielo y Dialnet, en el idioma español, trabajos de investigación que tienen como fuente el contexto de las TIC en el ámbito educativo.

Los criterios de inclusión de los artículos de investigación incluyeron: artículos resultados de investigación e indexación de Redalyc, Scielo y Dialnet; artículos de análisis y artículos de revisión teórica; los criterios de exclusión fueron: artículos editoriales y artículos no indexados. Las dimensiones analizadas fueron: Base de datos, Temática trabajada, publicaciones por país, tipo de estudio y diseño y publicaciones por revistas. El material bibliográfico recolectado se tabuló y codificó en el programa Excel y fue procesado estadísticamente por medio del programa estadístico Statistical Package for Social Science (SPSS), mediante análisis descriptivo de frecuencias de las variables y los resultados se presentan en figuras.

\section{RESULTADOS}

A continuación, se presentan a nivel descriptivo comparativo las principales dimensiones bibliométricos utilizados para cumplir con los objetivo de la investigación. En cuanto a la bases de datos de publicaciones, Redalyc, es la base de datos que registra un mayor número de publicaciones en el año 2020 (56\%) en comparación al año 2019 (28\%), en segundo lugar la revista Scielo (28\%) en comparación al año $2019(20 \%)$ y finalmente, Dialnet en el 2020 (I8\%) y en el 2019 (52\%) en relación a las publicaciones sobre las TIC en el contexto de la educación.

En cuanto a las temáticas trabajadas, la temática más tratada durante la pandemia, es la Educación básica (32\%) en comparación al 2019 (28\%); del mismo modo, la educación universitaria y la ciencia de la salud, tienen una producción durante la pandemia del (16\%) y (40\%) y (8\%) respectivamente en el 2019; la educación a la distancia en el 2020 (4\%) y en el 2019 (4\%), la educación tecnológica en el 2020 (8\%) y en el 23019 (4\%), finalmente, otros enfoques en el 2020 (24\%) y en el 2019 (16\%), en relación a las publicaciones sobre las TIC en el contexto de la educación.

En cuanto a los países de habla en español, que Ecuador es el país con mayor producción de revistas durante el 2020 (10\%) en comparación al (5\%) del 2019; España también tiene un aumento durante el 2020 (5\%) en comparación al 2019 (1\%); México durante el 2020 (3\%) disminuye su producción en comparación al 2019 (5\%); Colombia durante el 2020 (3\%) disminuye su producción en comparación al 2019 (6\%); Cuba durante el 2020 (0\%) disminuye su producción en comparación al 2019 (I\%); Chile durante el 2020 (I\%) disminuye su producción en comparación al 2019 (2\%);Bolivia durante el 2020 aumenta su producción (1\%) en comparación al 2019 (0\%); Venezuela durante el 2020 aumenta su producción (I\%) en comparación al 2019 (0\%); Argentina durante el 2020 (0\%) disminuye su producción en comparación al 2019 (I\%); Perú durante el 2020 (0\%) 
disminuye su producción en comparación al 2019 (3\%) y Paraguay durante el $2020(0 \%)$ disminuye su producción en comparación al 2019 (I\%), en relación a las publicaciones sobre las TIC en el contexto de la educación

En cuanto a las publicaciones por tipo y diseño de investigación, en cuanto al tipo de investigación, existe una relativa paridad entre los tipos cuantitativos producidos en el 2020 (48\%) y el 2019 (48\%); en cuanto al nivel cualitativo,; en el 2020 (37\%) disminuyo en comparación al 2019 (40\%) y en cuanto al tipo mixto, la producción del 2020 (I5\%) es mayor en comparación al 2019 (II\%); en relación a las publicaciones sobre las TIC en el contexto de la educación. En cuanto al diseño descriptivo del 2020 (28\%) disminuyo en comparación al 2019 (32\%); el diseño exploratorio descriptivo del 2020 (I2\%) aumento en comparación al 2019 (7\%); en cuanto al diseño de análisis del 2020 ( $16 \%)$ disminuyo en comparación a la del 2019 (28\%); en cuanto al correlacional (8\%) aumento en comparación al 2019 (\%); el exploratorio (0\%) disminuyo al del 2019 (2\%); en el diseño explicativo rexiste una paridad entre el 2020 (I5\%) y el 2019 (I5\%), en el diseño de casos en el 2020 (8\%) aumento en comparación al 2019 (4\%) y el diseño descriptivo explicativo (I $2 \%$ aumento en comparación al 2019 (3\%), en relación a las publicaciones sobre las TIC en el contexto de la educación.

En relación al número de artículos por revista indexada, existe paridad en el número de publicaciones realizadas en la revista Investigación, Desarrollo e Innovación público realizadas el 2020 (I2\%) en comparación al 2019 (I2\%); la revista ciencias humanísticas y sociales (I2\%) público en mayor número que en el 2019 (4\%); la revista ciencias e investigación en el 2020 (12\%) público en mayor número que en el 2019 (4\%); del mismo modo, la revista iberoamericana (8\%) público en mayor número que el en el 2019 (4\%), pese a ello, las publicaciones en el 2020, son muy exiguas en relación a las TIC en el contexto de la educación

Teniendo en cuenta que el objetivo de la presente investigación fue realizar una descripción comparativa de la producción de revistas en las bases de datos Scielo, Dialnet y Redalyc realizadas durante el 2019 y 2020, se puedo observar a través de dicho proceso comparativo, un aumento del interés por las temáticas relacionadas con la Investigación, Desarrollo e Innovación, con las Ciencias Humanísticas y Sociales y la ciencia y la investigación, pero aun dicho aumento de nivel de interés, resulta incipiente en la búsqueda de un fundamento teórico sólido y consistente relación a las TIC en el contexto de la educación en tiempos de pandemia.

\section{DISCUSIÓN}

A través de la comparación de la producción de artículos de investigación realizados entre los años 2019 y 2020 , se identificó que existe un mayor nivel de interés en ciertos campos de estudios como: la Investigación, Desarrollo e Innovación, con las Ciencias Humanísticas y Sociales y la ciencia y la investigación durante el 2020 , que se manifiesta en un mayor nivel de producción de artículos de investigación de estas temáticas que en el 2019, del mismo modo, se identificó que el mayor movimiento de producción se halla en el áreas de nivel básico como de nivel universitario; sin embargo, se evidencia que 
existe un interés creciente por temas de la educación virtual, la comunicación aplicada, conceptos que han empezado a generar líneas de investigación en las TIC enfocadas al contexto educativo en tiempos de pandemia.

\section{REFERENCIAS}

Alva, A. (20I5a,b). Los nuevos rostros de la desigualdad en el siglo XXI: la brecha digital. Revista Mexicana de Ciencias Políticas y Sociales, 60(223), 265-286, e-ISSN: 01851918. Recuperado de: http://dx.doi.org/10.1016/S0185-1918(15)72138-0

Barros-Bastidas, C., \& Gebera, O. T. (2020). Training in research and its incidence in the scientific production of teachers in education of a public university of Ecuador. Publicaciones de La Facultad de Educacion y Humanidades Del Campus de Melilla, 50(2), 167-I85. https://doi.org/I0.30827/publicaciones.v50i2.13952

(2000). La era de la información. Revista La sociedad red. En: 2da ed. Madrid, España: Alianza.

Cabero, J., (1998). Impacto de las nuevas tecnologías de la información y la comunicación en las organizaciones educativas. Granada: Revista Editorial Universitario, Pp. 197206.

Cinque, M. (2016). Lost in translation. Soft skills development in European countries. Tuning Journal For Higher Education, 3(2), 389-427. Recuperado de: doi:http:// dx.doi.org/I0.18543/tjhe-3(2)-2016pp389-427

Cueva, J.., García, A., \& Martínez, O. (2019). El conectivismo y las TIC: Un paradigma que impacta el proceso enseñanza aprendizaje. Revista Scientific, 4(I4), 205-227, eISSN: 2542-2987. Recuperado de: https://doi.org/I0.29394/Scientific.issn.25422987.2019.4.14.10.205-227

Fiszbein, A., C. Cosentino, y Cumsille B. (2016). El desafío del desarrollo de habilidades en América Latina: Un diagnóstico de los problemas y soluciones de política pública. Washington, DC: Diálogo Interamericano-Mathematica Policy Research.

Fundación Wiesse (2020). El gran reto de la educación virtual en tiempos de pandemia. Edit. Newsletter. Recuperado de: https://www.fundacionwiese.org/blog/es/el-granreto-de-la-educacion-virtual-en-tiempos-de-pandemia/

Ippolito-Shepherd, J.; Cerqueira, M. T. (2003). Las escuelas promotoras de la salud en las Américas: una iniciativa regional. Washington, DC: Organización Panamericana de Salud. Recuperado de: ftp://ftp.fao.org/docrep/fao/006/j0243m/j0243m03.pdf

M. Cinque (2015). Comparative analysis on the state of the art of Soft Skill identification and training in Europe and some Third Countries.," New perspectives in teaching.

Mangrulkar, L.; Whitman, C. V.; Posner, M. (200I). Enfoque de habilidades para la vida para un desarrollo saludable de niños y adolescentes. Washington, DC: Organización Panamericana de Salud. Recuperado de: https://convivencia.files.wordpress.com/2008/I I/habilidades200 I oms65p.pdf

Matus, O., \& Gutiérrez A. (20I5). Habilidades Blandas: Una ventaja competitiva 2015. Recuperado https://convivencia.files.wordpress.com/2008/I I/habilidades200 I oms65p.pdf 
Maya, M. \& Orellana, B. (2017). Desarrollo de soft skills una alternativa a la escasez de talento humano. INNOVA Research Journal, I(I2), 59-76.

Mujica, J. (2015, Enero). ¿Qué son las habilidades blandas y cómo se aprenden? Recuperado de: https://educrea.cl/wpcontent/uploads/2016/02/DOC-habilidadesblandas.pdf?×65104

Ruiz, L. (2007). Formación integral: desarrollo intelectual, emocional, social y ético de los estudiantes. Revista Universidad de Sonora, 19, II-13.

Pinedo, I. (2017). Programa de Capacitación Docente: Tecnologías del Aprendizaje y del Conocimiento - TACs. Trabajo de Grado. Panamá: Universidad Especializada de las Américas. Recuperado de: http://repositorio2.udelas.ac.pa/handle/I 23456789/32

Universidad de Piura (2020, 5 de abril). La educación virtual en tiempos de pandemia. Consultado el 07 de diciembre de 2020. http://udep.edu.pe/hoy/2020/laeducacion-virtual-en-tiempos-de-pandemia/

World Health Organization (2003). Skills for Health. Recuperado de:http://www.who.int/school_youth_health/media/en/sch_skills4health_03.pdf 